\title{
UM OLHAR PARA A MATEMÁTICA PRESENTE NO AMBIENTE DA ELETROTÉCNICA: CONTRIBUIÇÕES PARA A EDUCAÇÃO PROFISSIONAL
}

\author{
E. S. COSTA \\ Instituto Federal de Educação, Ciência e Tecnologia da Bahia - IFBA \\ edcarloscosta@ifba.edu.br ${ }^{*}$
}

Artigo submetido em outubro/2013 e aceito em dezembro/2013

DOI: $10.15628 /$ rbept.2013.3495

\section{RESUMO}

Este trabalho trata-se de uma pesquisa embrionária que tem como proposta apontar alguns caminhos, ainda em construção, ao identificar a matemática inerente aos mecanismos da eletrotécnica e buscar, por meio da etnomatemática, uma prática pedagógica mais dinâmica e significativa, que proporciona condições para que o educando construa relações e possa solucionar problemas do cotidiano vivenciado pelos profissionais da área elétrica. Em alguns conteúdos esta relação já pôde ser identificada, e a metodologia aplicada visou estabelecer uma relação entre fenômenos naturais observados e vividos pelo grupo de estudantes e os conteúdos teóricos e práticos do curso de eletrotécnica. Foi possível observar que após a correlação dos conceitos científicos com a vida social e cultural dos estudantes, houve uma melhor compreensão do conteúdo trabalhado, que denota a percepção do conhecimento como uma produção do pensamento pela qual se apreendem e se representam as relações que constituem e estruturam a realidade.

PALAVRAS-CHAVE: Etnomatemática, Educação Profissional, Eletrotécnica.

\section{A LOOK FOR MATH THIS IN THE ELECTROTECHNICAL ENVIRONMENT: CONTRIBUTIONS FOR PROFESSIONAL EDUCATION}

\begin{abstract}
This work it is an embryonic research that has the purpose to point out some ways, still under construction, to identify the mathematics inherent IN mechanisms of electrical engineering and seek, through Ethnomathematics, a more dynamic and meaningful pedagogical practice, which provides conditions for that the student build relationships and can solve everyday problems experienced by professionals in the electrical area. In some content this relationship might already be identified, and the methodology aimed at establishing a
\end{abstract}

relationship between natural phenomena observed and experienced by the group of students and the theoretical and practical content of the course of electrical engineering. It was observed that after the correlation of scientific concepts to the social and cultural life of the students, there was a better understanding of the content worked, which denotes the perception of knowledge as a production of thought by which we perceive and represent the relationships that constitute and structured the reality.

KEYWORDS: Ethnomathematics, Professional Education, Electrical Engineering. 


\section{APRESENTAÇÃO}

A matemática é considerada por muitos indivíduos como uma disciplina com grau de dificuldade elevado, devido principalmente ao fato dos estudantes não conseguirem estabelecer relações entre os conteúdos estudados e as situações e problemas vividos em seu cotidiano. Os educadores também vivenciam este problema, a dificuldade de ensinar ciências exatas hoje em dia é uma realidade nas escolas de ensino médio, assim como, nos cursos de educação profissional.

As operações aritméticas, definições, teoremas, procedimentos algébricos, geralmente, não são apresentados de maneira que se compreendam os porquês e as justificativas para sua utilização. Este fato faz com que não haja uma maior aproximação, por parte dos estudantes, destes temas, acarretando um elevado índice de reprovação nas instituições de ensino.

Percebe-se que a principal complicação encontrada pelos educadores, assim como, pelos educandos é a associação dos conteúdos abordados em matemática com sua real aplicação nas práticas cotidianas.

Notamos que, a situação torna-se ainda mais preocupante nos cursos de formação profissional. A compreensão dos assuntos específicos de alguns cursos profissionalizantes exige que os estudantes dominem as ferramentas matemáticas presentes nesses conteúdos. Observando o currículo do curso de eletrotécnica, é possível perceber em muitos componentes curriculares, assim como, nas práticas do profissional em elétrica, a necessidade das ferramentas matemáticas para a elucidação dos assuntos tratados.

Observa-se dessa maneira a necessidade de tornar significativo o conhecimento matemático necessário à formação daqueles futuros eletrotécnicos, a partir da identificação o papel da matemática como estruturante do pensamento físico, do mundo material, uma matemática própria da prática do eletrotécnico.

Em meio às dificuldades de definição do caminho para o alcance dos objetivos optamos por mostrar o percurso escolhido - enquanto docente - para compreensão da matemática inerente ao ambiente profissional mencionado, acessível aos meus estudantes, que viam conhecimentos fragmentados e desconectados uns dos outros.

A matemática, ali, surgia como uma possibilidade de encantar-se e perceber o significado do que estudavam, estabelecendo uma rede de relações. A matemática e as suas relações. A matemática inerente ao campo da Eletrotécnica.

\section{A ETNOMATEMÁTICA INERENTE AO AMBIENTE DA ELETROTÉCNICA}

A palavra etnomatemática é composta por três principais raízes: etno, compreendendo os diversos ambientes (o social, o cultural, o natural, o imaginário); matema, significando explicar, entender, ensinar, lidar com; e tica, que advém da palavra grega tecné, que se refere a artes, técnicas, maneiras. Assim, segundo D’Ambrosio (2008), "a etnomatemática significa o conjunto de artes, técnicas de explicar e de entender, de lidar com o ambiente social, cultural e natural, desenvolvido por distintos grupos culturais".

Assim sendo, o campo da Eletrotécnica possui uma matemática inerente às suas questões e práticas, à qual contempla suas necessidades resolver sistemas de equações, de medir 
resistência, potência, tensão e corrente elétrica em componentes eletro-eletrônicos, entender e calcular frequências, períodos e ciclos de sinais alternados, comparação entre ondas senoidais de tensão elétrica, realizar transformações com números complexos, calcular defasagem entre sinais de tensão e corrente, calcular matrizes provenientes de circuitos elétricos, esboçar gráficos de funções de correntes e tensões contínuas e alternadas, calcular tensão de curto circuito, entre outras artes, técnicas de explicar e compreender o comportamento de componentes e circuitos elétricos, imprescindíveis nesse ambiente.

Diante de tal cenário, notamos que, possibilitar que o estudante de Eletrotécnica notasse uma Etnomatemática dentro do seu espaço profissional, colaboraria com sua aprendizagem e esta se tornaria mais significativa na construção da sua educação profissional.

A proposta desse trabalho é apontar alguns caminhos que ainda estamos construindo, ao identificar a matemática inerente nos mecanismos da eletrotécnica e buscar, por meio da etnomatemática, uma prática pedagógica mais dinâmica e significativa, proporcionando condições para que o educando, através das associações feitas com o conhecimento matemático que ele traz com a matemática que emerge daquele espaço, sinta-se seguro de construir as relações e solucionar problemas do cotidiano vivenciado pelos profissionais da área da elétrica.

\section{A MOTIVAÇÃO}

Enquanto Engenheiro Eletricista e ex-estudante do curso de Eletrotécnica percebo que, a metodologia tradicionalmente utilizada e, ainda hoje aplicada, no ensino da Elétrica é um dos principais entraves no processo de aprendizagem do estudante. Percebe-se a existência de uma lacuna entre, o que se aprende no curso e a matemática inerente ao aprendizado dos conteúdos específicos da eletrotécnica. Acarreta, dessa maneira, uma formação mecanicista e fragmentada, na qual o estudante necessita memorizar um número excessivo de equações e métodos, sem fazer relação alguma, entre estes conteúdos e a sua real importância na carreira, como profissional da elétrica. Ou seja, não se desenvolve uma visão crítica da realidade.

Um importante componente da etnomatemática é a possibilidade de uma visão crítica da realidade, utilizando instrumentos da natureza matemática. Análise comparativa de preços, de compras, de orçamento, proporcionam um excelente material pedagógico. (D'AMBROSIO, 2007, p.23)

Outro fato observado, enquanto professor de Eletrotécnica do IFBA, é que existe um alto nível de reprovação e desistência de disciplinas específicas da elétrica, principalmente nos primeiros anos do curso. Um dos fatores que também acarretam estas reprovações é o descompasso entre os assuntos abordados em elétrica, que utilizam matemáticas específicas, os quais ainda não foram apresentados pelos professores de física e matemática. $O$ estudante não consegue notar relações e entende como um novo conteúdo a ser estudado. Este fato causa, na maioria das vezes, um retardo no aprofundamento e avanço dos conteúdos específicos do curso de elétrica, já que os estudantes apresentam dificuldades de estabelecer a correlação entre os componentes curriculares.

Estabelecer relações entre o que se aprende nas escolas e a vida cotidiana também é uma das propostas desta pesquisa e buscou-se através da etnomatemática criar estes caminhos, por 
acreditar que este tipo de prática potencializa a capacidade de aprendizado dos estudantes, conforme assinala Gerdes (2007), a "Etnomatemática mostra que uma condição para que a escola contribua para a realização do potencial de cada criança, reside na integração e incorporação dos conhecimentos matemáticos que a criança aprende fora da escola". Mesmo que os conhecimentos que ela possui sejam considerados muito espontâneos e/ou informais. Para este mesmo autor, a motivação para os estudos de uma criança esta relacionada à identificação que ela possa fazer do saber escolar e sua vivência cotidiana. E para que isso se concretize, pode incorporar-se no currículo elementos que pertencem ao ambiente sócio-cultural dos professores e, principalmente, dos estudantes, possibilitando o uso desses elementos em sala de aula.

Apesar do público alvo da pesquisa não se tratar de crianças, a aproximação feita com os conteúdos, conforme mencionado anteriormente, também pode ser aplicada. Vale ressaltar que, a proposta do trabalho não é substituir a matemática acadêmica pela etnomatemática, mas sim compreender a possibilidade de diálogo entre estas. Pois é de extrema importância para a incorporação dos conteúdos que os estudantes possam fazer associações do que é ensinado em sala de aula com as práticas matemáticas de seu cotidiano. No nosso caso, as práticas matemáticas provenientes do trabalho como técnico em elétrica. $O$ que podemos chamar de ensinar/aprender sob a perspectiva da etnomatemática.

Ensinar sob uma perspectiva etnomatemática é um modo de promover reformas no ensino, engajando os estudantes na descoberta da matemática de seus cotidianos, de seus pais e amigos de muitas culturas. A perspectiva etnomatemática traz interesse, excitação e relatividade para os estudantes, que serão mais motivados como estudantes de matemática em geral. (WENGER, 1998 apud SANTOS, 2002)

Algumas instituições de ensino já incorporaram esta prática em seus currículos por percebe a relevância que a etnomatemática tem no ensino da matemática. Isso é possível ser evidenciado nas palavras de Jaime Carvalho e Silva quando apresenta, de maneira muito apropriada, os currículos das escolas portuguesas na disciplina de Matemática das escolas secundárias de ensino artístico, no prefácio do livro "Etnomatemática: Reflexões sobre Matemática e Diversidade Cultural" de Paulus Guerdes, dizendo que:

"Devem ser utilizados exemplos concretos como, por exemplo, barras de tapetes de Arraiolos, azulejos, mosaicos (como os de Conímbriga), padrões geométricos africanos (sipatsi, lusona, etc.), geometria de igrejas, monumentos conhecidos (Cubo da Ribeira, Monumento ao empresário, monumento de Condeixa à autoestrada, esculturas das áreas de serviço das auto-estradas, etc.) directamente ou através de fotografias." (SILVA, 2007, p.12)

Essa prática adotada pelas escolas portuguesas é um bom exemplo a ser seguido nos cursos de Educação Profissional, no nosso caso, no curso de Eletrotécnica, devido ao fato deste apresentar um número significativo de componentes curriculares e de práticas profissionais, que se fundamentam em teoremas e procedimentos matemáticos próprios da área. 


\section{ELETROTÉCNICA: FONTE DA QUAL EMANA UMA MATEMÁTICA}

É possível observar na atualidade, as diversas formas de cobranças vividas pelos educadores no que diz respeito ao desenvolvimento de atividades que venham a convergir na direção ao respeito à diversidade sociocultural e à aprendizagem dos educandos. Exigindo assim dos educadores uma maior dedicação e pesquisa sobre novas maneiras e alternativas de ensino para a elaboração destas atividades. Segundo Santos (2002), "a preocupação por ter algo pronto, na qualidade de uma técnica ou um método, que possa aplicar de forma imediata tornou-se, de algum modo, inerente ao ato de ensinar nos moldes tradicionais".

Baseado neste contexto percebemos a etnomtemática como detentora de relações inclusivas entre educadores e educandos, assim como, auxiliadora no desenvolvimento de atividades que confluam em direção ao respeito aos contextos culturais/socioculturais diferentes.

(...) defendemos a etnomatemática não como um método de ensino em si, mas sim como detentora de relações inclusivas entre professores e alunos e das diversas formas de conhecer presentes em contextos culturais/socioculturais diferentes. Isso porque, mais importante que a mudança/indicação de métodos e técnicas, é a necessidade de haver/desenvolver questionamentos e reflexões sobre as nossas próprias práticas, condutas e idéias (VIEIRA, 1999 apud SANTOS, 2002).

Assim, como disse o autor, a etnomatemática também nos auxiliará na reflexão sobre condutas e práticas que amenizem os problemas encontrados na transmissão/aprendizado dos conhecimentos inerentes ao curso de eletrotécnica. Portanto, a escolha da proposta metodológica de ensino/aprendizagem ocorreu a partir da percepção de problemas identificados no curso de elétrica. Trata-se da metodologia da problematização, nesta metodologia os problemas são extraídos da realidade pela observação realizada pelos estudantes.

Segundo Berbel (1998), a primeira referência para essa metodologia é o Método do Arco, de Charles Maguerez, que se divide basicamente em cinco etapas que se desenvolvem a partir da realidade ou um recorte da realidade. São elas: Observação da Realidade; Pontos-Chave, Teorização; Hipóteses de Solução e Aplicação à Realidade (prática).

A primeira etapa refere-se à observação da realidade social, os estudantes foram incentivados a observar atentamente os assuntos que eles mais possuíam dúvidas e dificuldade de aprendizado e assim registrar sistematizadamente o que identificaram. Relatando as principais dificuldades, carências e discrepâncias. Na segunda etapa, que é a dos Pontos-Chaves, os estudantes foram levados a refletir primeiramente sobre as possíveis causas da existência dos problemas mencionados.

A partir dessa análise reflexiva, os estudantes foram estimulados a sintetizar possibilidades de resolução dos pontos essenciais que deverão ser estudados, para compreendê-los mais profundamente e encontrar formas de interferir na realidade para solucioná-lo ou desencadear passos nessa direção. A partir desses dados, iniciamos a terceira etapa que é a da teorização. Esta é a etapa do estudo, da investigação propriamente dita. Os estudantes foram separados em grupos e se organizaram tecnicamente para buscar, com a ajuda dos professores, as informações propedêuticas que necessitavam sobre cada problema levantado. A quarta etapa é a das hipóteses 
de solução, ou seja, após todo o estudo realizado os estudantes deveriam responder a perguntas como: O que precisa acontecer para que o problema seja solucionado? O que precisa ser providenciado? O que pode realmente ser feito? E mais uma vez, foram encorajados e realizar críticas e criativamente, com ajuda do professor, elaborar as possíveis soluções.

Por fim, realizamos a quinta e última etapa, que é a da Aplicação à Realidade. Esta etapa da Metodologia da Problematização ultrapassa o exercício intelectual. Ela deve ser feita de acordo com a criatividade dos estudantes e professores, sofrendo influência direta de suas práticas e vivências. Nas palavras de Berbel:

“[...], pois as decisões tomadas deverão ser executadas ou encaminhadas. Nesse momento, o componente social e político está mais presente. A prática que corresponde a esta etapa implica num compromisso dos alunos com o seu meio. Do meio observaram os problemas e para o meio levarão uma resposta de seus estudos, visando transformá-lo em algum grau" (BERBEL, 1996, p.8-9).

Elegemos aqui o tema corrente alternada para exemplificar a existência de uma matemática própria do ambiente da elétrica. Pois para que posamos afirmar que existe uma etnomatemática própria a este ambiente, faz necessário perceber a matemática que emerge desta prática profissional. A escolha deste tema se dá também por se tratar de um conteúdo de suma importância para o curso de eletrotécnica.

Durante este curso, o estudante aprende a analisar e medir um sinal alternado seja ele monofásico, como é o caso de medições residenciais, ou trifásico como é o caso da maioria das grandes indústrias. Ensinar ao estudante como realizar uma medição corretamente, assim como entender o comportamento de um sinal elétrico é de suma importância, pois ao longo de sua carreira como eletrotécnico ele (a) estará praticando isso toda vez que for necessário realizar intervenções em circuitos elétricos, em linhas de transmissão, ou mesmo na realização de projetos elétricos e dimensionamento de equipamentos de proteção coletiva e/ou individual, como por exemplo, estabelecer os critérios para a indicação de equipamentos de proteção individual (EPI's).

Nestas práticas profissionais são utilizados equipamentos específicos para realizar estas medições e testes (amperímetros, voltímetros, frequencímetros e outros), assim é de extrema importância que o estudante de elétrica saiba o funcionamento destes equipamentos e quais são os cálculos "realizados" por estes internamente para inferir determinada grandeza. Além de está preparado para realizar estas operações na ausência de um determinado equipamento de medição ou no caso de identificar falhas nestes. Baseado nisso, saber analisar um sinal alternado é uma prática desta área que exige e emerge uma matemática específica.

Para o melhor entendimento desta prática, primeiramente é importante explicitar alguns conceitos. O primeiro deles é o de corrente elétrica. Define-se como corrente elétrica o deslocamento de cargas elétricas dentro de um condutor, quando existe uma diferença de potencial elétrico entre as extremidades deste. De acordo com Pietrocola essa corrente pode ser contínua:

[...] representada pela sigla CC ou DC (do inglês direct current), quando o seu sentido permanece constante ao longo do tempo. Esse tipo de corrente é produzida, principalmente, por pilhas, baterias e células fotovoltaicas. A corrente alternada representada por CA ou AC (do inglês alternating current), é um fluxo 
oscilante de cargas que ora se movimenta em um sentido,ora em outro. (PIETROCOLA, 2013, p.29)

A forma de onda da corrente alternada senoidal pode ser representada no domínio temporal por:

$I(t)=\operatorname{Ip} \cdot \operatorname{Sen}(\omega t+\theta)$

Onde:

Ip: Corrente de pico (valor máximo da corrente) $[\mathrm{A}]$;

$\omega$ : Frequência angular (mede quantos radianos a corrente se deslocou em 1s) [rad/s];

$\omega=2 \pi f \quad$ ou $\quad \omega=\frac{2 \pi}{T}$

f: freqüência da rede elétrica (60 Hz no Brasil) [Hz];

$T$ : Período (tempo necessário para se completar um ciclo) [s].

Analisando esta equação, percebemos que emana deste conteúdo da eletrotécnica o assunto estudado na matemática sobre funções trigonométricas, que são funções angulares importantes no estudo dos triângulos e na modelação de fenômenos periódicos. Como é o caso do sinal da corrente ou tensão alternada.

Para ilustrar, representamos abaixo a forma de onda e o diagrama fasorial de uma tensão alternada (CA), onde é possível observar o uso de mais um termo usado na matemática da prática do eletrotécnico. Trata-se do uso do ciclo trigonométrico para representar os ângulos de fase iniciais $(\theta)$ e o sentido de giro da freqüência angular $(\omega)$. Na matemática, o círculo trigonométrico, círculo unitário, ou círculo goniométrico é um círculo cujo centro está localizado na origem do plano cartesiano e seu raio mede 1 . É usado no estudo de funções trigonométricas como seno, cosseno e tangente. Portanto pode ser aplicado na eletrotécnica, pois a equação que descreve um sinal alternado pode ser conceituada matematicamente como uma função trigonométrica.
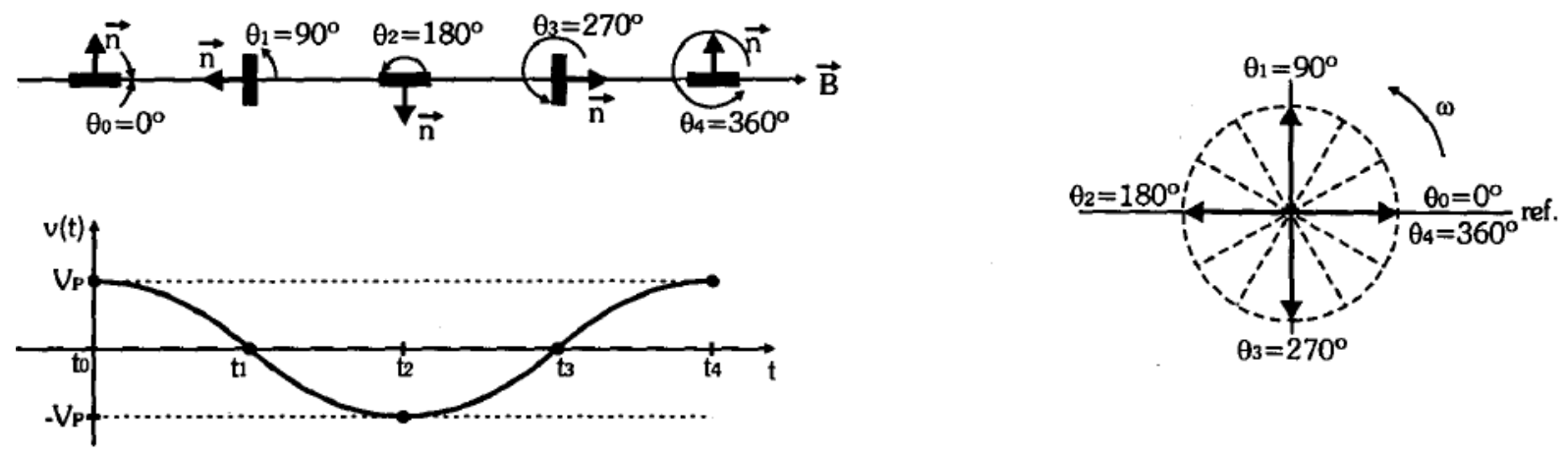

a)

b)

Figura 1 - Forma de onda da tensão alternada - a) forma fasorial; b) diagrama fasorial.

Segundo Pietrocola (2013), novas perspectivas para os objetivos da educação formal vêm se desenvolvendo há algumas décadas, centrando no papel dos conteúdos ensinados para a vida em sociedade e nos processos de organização e construção do conhecimento pelo próprio aluno.

Alguns pesquisadores sobre etnomatemática também concordam com Pietrocola, por exemplo, para Santos (2002) a etnomatemática "não se limita a ensinar matemática aos educandos 
e educandas, mas sim, de alguma forma, tendo como norte as diversas etnomatemáticas, almeja alfabetizá-los matematicamente relevando suas formas matemáticas próprias, suas realidades e anseios, seus meios socioculturais e culturais".

Em concordância com o que defendemos, continua sua argumentação dizendo que:

Vale ressaltar é nessa direção de encaminhamentos que, de algum modo, poderão emergir dos alunos suas maneiras próprias de quantificar, de inferir, de resolver problemas, de entender a sua realidade, a sua matemática, isto é, a sua etnomatemática. (SANTOS, 2002, s/p)

Assim, ao invés de utilizar a forma convencional de explicação do ciclo trigonométrico, visto que o entendimento deste é de extrema necessidade para a prática do profissional de eletrotécnica, os estudantes foram encorajados a explorar possibilidades, inventar soluções, alternativas e colaborar para a construção do conhecimento. Para tal buscou-se realizar a construção do ciclo trigonométrico utilizando associações com os fenômenos naturais conhecidos e vividos pelos próprios estudantes.

A construção junto com os estudantes faz com que esses não tenham apenas o mero acesso à informação, mais faça parte da construção desta, não restringindo a possibilidade de nela atuar. A primeira associação foi feita com a ideia de ciclos e períodos (variáveis importantes à compreensão do comportamento da corrente alternada). Desta forma, buscou-se associar o ciclo do dia, que compreende a trajetória do sol no círculo celeste, com o sentido de giro da freqüência angular (que é o mesmo do ciclo trigonométrico, anti-horário).

Ainda buscando a interdisciplinaridade com os estudos de geografia, outra associação foi feita entre o círculo trigonométrico e os pontos cardeais. Ao desenharmos os pontos cardeais associando o inicio do ciclo trigonométrico $\left(0^{\circ}\right)$ com o Leste, e seguindo o sentido do ciclo, temos o Norte comparado à posição de $90^{\circ}(\pi / 2)$, o Oeste a $180^{\circ}(\pi)$, o Sul a $270^{\circ}(3 \pi / 2)$, voltando outra vez para o Leste completando o ciclo de $360^{\circ}(2 \pi)$. Que na nossa compreensão seria o ciclo completo de rotação realizado em 24 horas pelo sol.

Esta dinâmica foi feita com a sala arrumada em semi-círculo e com a participação dos estudantes em todo processo, de modo que se tornou possível a visualização mais aproximada do conceito científico a partir de um fenômeno natural. Como pode ser observado na figura abaixo:

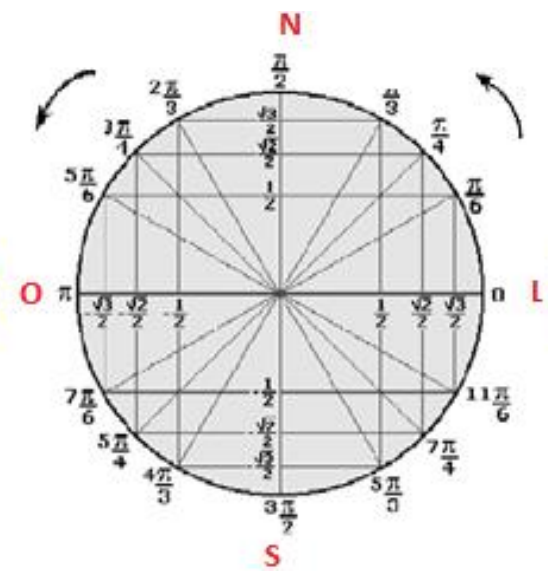

Figura 2 - Círculo trigonométrico x pontos cardeais 
Esse processo de investigação e associação do conceito científico com acontecimentos do cotidiano nos remete a um dos pensamentos de Paulo Freire, que diz:

[...] conceber a prática de ensino como um processo de permanente investigação significa assumir o posicionamento epistemológico em que o educando é o sujeito de seu conhecimento, estando sua aprendizagem associada a um processo constante de pesquisa sobre sua realidade. Em outras palavras, significa não distanciar a prática educativa e do exercício da curiosidade epistemológica dos educandos. (FREIRE, 1997 apud SAUL, 2012).

Ainda sobre o assunto de corrente alternada, podemos identificar que outro conteúdo dessa matemática que emana das práticas deste profissional, e é utilizada para explicar este fenômeno, é a representação da corrente ou da tensão elétrica através dos números complexos e representações fasoriais.

Segundo Markus (2004), um sinal alternado senoidal (ou cossenoidal) pode ser convertido diretamente nas representações fasorias e complexas. Assim, temos:

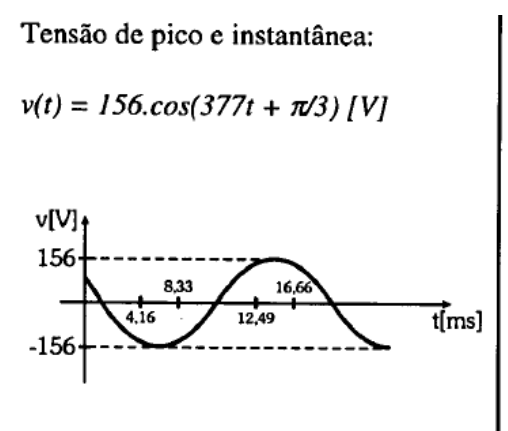

a)

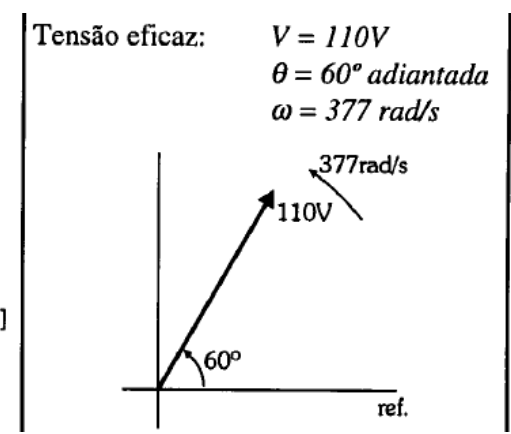

b)

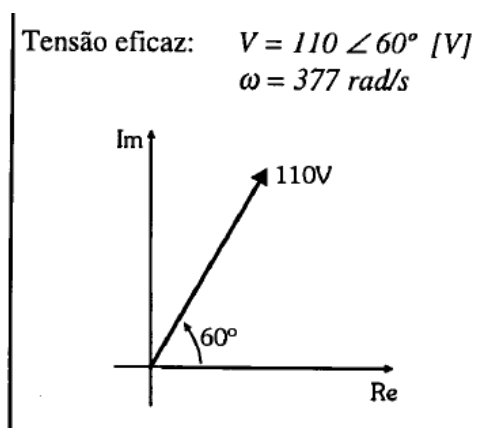

c)

Figura 3: Representações de um sinal alternado - a) tensão de pico e instantânea; b) tensão eficaz; c) tensão eficaz.

$\mathrm{V}=110 \angle 60^{\circ}[\mathrm{V}]-$ Representação na Forma Polar de um sinal alternado.

$V=55+95,26 j[v]-$ Representação na Forma Retangular de um sinal alternado.

Esta última equação é originária da expressão $V=V p \cdot \cos (\theta)+V p \cdot \operatorname{sen}(\theta) j$, onde

$V p=110[v]$ e $\theta=60^{\circ}, \operatorname{assim} V=110 \cdot \cos (60)+110 \operatorname{sen}(60) j$, resultando na expressão:

$\mathrm{V}=55+95,26 \mathrm{j}[\mathrm{v}] \longleftrightarrow \mathrm{Z}=\mathrm{a}+\mathrm{bi}$

O Técnico em Eletrotécnica em sua vida cotidiana necessita realizar cálculos de grandezas elétricas para, por exemplo, dimensionar equipamentos e instrumentos elétricos (sejam eles de proteção, identificação, automação, etc). Assim, é necessário que este entenda o funcionamento dos componentes elétricos, assim como, o comportamento destas grandezas nos circuitos elétricos. E uma das formas de analisarmos estas grandezas é observarmos o comportamento desta através das equações matemáticas que as descrevem.

Por exemplo, observando a equação $V=55+95,26$ j, identificamos aí o uso dos números complexos $(Z=a+b i)$ para explicitar o comportamento de um sinal alternado de tensão elétrica, ou seja, a matemática emergindo da prática do profissional que trabalha com eletricidade. 
Na disciplina de matemática, o coeficiente $a$ é a parte real de $Z$, representada por $\operatorname{Re}(Z)$, e o coeficiente $b$ é a parte imaginária de $Z$, representada por $\operatorname{Im}(Z)$. No caso do sinal elétrico (tensão alternada) o $i$, que representa a parte imaginária, é substituído por $j$, a fim de não confundir com a letra i também utilizada para representar corrente elétrica.

Para D’Ambrosio (2007), “a disciplina denominada matemática é uma etnomatemática que se originou e se desenvolveu na Europa, tendo recebido algumas contribuições das civilizações indianas e islâmicas".

No ambiente da eletrotécnica, outras práticas e assuntos como ensaios e manutenção de máquinas e equipamentos elétricos, testes e dimensionamento de componentes eletrônicos, escolha de métodos para cálculos de iluminação de ambiente, sinalização e simbologia de equipamentos de instalações elétricas, análise do comportamento de equipamentos na geração, transmissão e distribuição de energia elétrica, procedimentos de interligação de linhas de transmissão, análise sobre corrente e corrente de curto-circuito, projetos elétricos residenciais e industriais, aquisição, implantação e teste em elementos de automação, dentre muitas outras práticas se fazem presentes. Posteriormente, com o avanço da pesquisa, estas práticas serão estudadas minuciosamente para que se identifique a matemática que, como já demonstramos com os exemplos dados, está presente e emana desta área de atuação profissional.

\section{CAMINHOS A PERCORRER}

Mesmo se tratando de uma pesquisa embrionária, foi possível observar que após a correlação dos conceitos científicos com a vida social e cultural dos estudantes, estes absorveram melhor os conteúdos trabalhados, compreendendo o conhecimento como uma produção do pensamento pela qual se apreende e se representam as relações que constituem e estruturam a realidade.

Analisando minha trajetória enquanto ex-estudante de Eletrotécnica e Engenharia Elétrica e observando os relatos dos estudantes com relação às dificuldades encontradas para assimilar os conteúdos do curso, foi possível diagnosticar que os métodos tradicionais que vem sendo utilizados ao longo dos anos para ensino das ciências exatas já são declarados como grandes formadores de barreiras sociais, que na educação se reflete no afastamento do acesso em cursos de graduação e/ou pós graduação que necessitem dos conhecimentos das áreas de exatas. Diante desses fatos, faz-se necessários repensar as práticas educacionais correntes e buscar amparo também em metodologias de ensino que se pautem na valorização dos conceitos matemáticos informais construídos por determinados grupos fora do contexto institucional, buscando despertar nos estudantes suas potencialidades que resultam num aprendizado mais consistente e dinâmico.

Com este trabalho foi possível perceber que existe uma matemática própria utilizada na prática da área elétrica para explicar os conceitos e fenômenos existentes. Com o avanço da pesquisa, será possível identificar e apontar outros assuntos matemáticos, que amparam os teoremas e procedimentos utilizados nesta área. A fim de possibilitar que o estudante de Eletrotécnica estabeleça uma melhor relação com o que se compreende em matemática com os conteúdos específicos do curso de Eletrotécnica. 


\section{REFERÊNCIAS}

1. BERBEL, N. A. N. Metodologia da Problematização no Ensino Superior e sua contribuição para o plano da praxis. Semina : v.17, n. esp., p.7-17, 1996.

2. A problematização e a aprendizagem baseada em problemas: diferentes termos ou diferentes caminhos? Scielo. 1998. Disponível em< http://www.scielo.br/pdf/icse/v2n2/08>. Acesso em: 25 Jan. 2015.

3. BOYLESTAD, R. Introdução à análise de circuitos. 10. Ed. São Paulo: Pearson Prentice Hall, 2004.

4. D’AMBRÓSIO, U. Etnomatemática: Elo entre as tradições e a modernidade. 2. Ed. Belo Horizonte: Autêntica, 2007.

5. . O programa Etnomatemática: uma síntese. Acta Scientiae, Canoas, n.1, p.7-16, 2008.

6. FREIRE, P. Educação e Mudança. 12. Ed. Rio de Janeiro: Paz e Terra, 1979.

7. GADOTTI, M. Concepção dialética da educação. São Paulo: Cortez, 1995.

8. GERDES, P. Etnomatemática: Reflexões sobre matemática e diversidade cultural. Ribeirão: Húmus, 2007.

9. MARKUS, O. Circuitos elétricos: Corrente contínua e Corrente Alternada. 9. Ed. São Paulo: Érica, 2004.

10. MORIN, E. Os sete saberes necessários à educação do futuro. São Paulo: Cortez, 2001.

11. PETROCOLA, M. Física- conceitos e contextos. São Paulo: FTD, 2013. (vol. 3)

12. RICIERE, A.P. Arqueologia Matemática: A origem da Matemática nas civilizações antigas. São Paulo: Parma, 1991.

13. SANTOS, B. P. A etnomatemática e suas possibilidades pedagógicas: algumas indicações. São Paulo, 2002. Disponível em: < http://www.mat.uc.pt/ mat1287/texto/etnomatematica.htm>. Acesso em: 17 jan. 2015.

14. SAUL, A. M / INSTITUTO PAULO FREOIRE DE ESPAÑA. Uma prática docente inspirada no "jeito de ser docente" de Paulo Freire. Rizoma Freiriano. Disponível em < http://www.rizomafreireano.org/index.php/uma-pratica-docente-inspirada-no-jeito-de-ser-docente-de-paulofreire-ana-maria-saul >. Acesso em: 13 jan. 2015.

15. SILVA, J. C. Prefácio. In: GERDES, P. Etnomatemática: Reflexões sobre matemática e diversidade cultural. Ribeirão: Húmus, 2007. 